\title{
Methyl Mercury Injury to CNS: Mitochondria at the Core of the Matter?
}

\author{
Maria Rosaria Carratù ${ }^{1 *}$ and Anna Signorile ${ }^{2}$ \\ ${ }^{1}$ Department of Biomedical Sciences and Human Oncology, University of Bari, Italy \\ ${ }^{2}$ Department of Basic Medical Sciences, Neurosciences and Sense Organs, University of Bari, Italy
}

Submission: October 10, 2015; Published: November 17, 2015

*Corresponding author: Prof. Maria Rosaria Carratù, MD, Department of Biomedical Sciences and Human Oncology, University of Bari, Policlinico, Piazza Giulio Cesare 11, 70124 Bari, Italy, Tel. no: ++39 080 5478455; Email: mariarosaria.carratu@uniba.it

\begin{abstract}
Methyl-mercury (MeHg) is one of the most hazardous environmental pollutants of great concern to public health and regulatory agencies because of its primary toxicity to the human central nervous system. The major source of MeHg exposure to the general population is through consumption of contaminated fish and other food products. MeHg, absorbed from the gastrointestinal tract, is easily transported across the blood-brain barrier (BBB). Cysteine-facilitated transport of MeHg into the brain has been demonstrated, and in particular a neutral amino acid transport system capable of mediating MeHg-cysteine uptake has been identified in astrocytes where MeHg accumulation induces cell swelling and inhibition of glutamate uptake. Elevation of glutamate levels in the extracellular space may, in turn, trigger or accelerate processes of excitotoxic neuro degeneration. The rising of extracellular glutamate levels is responsible for the sustained activation of glutamate receptors, hence enhancing $\mathrm{Na}^{+}$influx and $\mathrm{Ca}^{2+}$ release from intracellular organelles that may trigger a biochemical cascade which promotes the reactive oxygen species (ROS) production. In this scenario, mitochondria may play a crucial role, as these organelles act as a buffer against cytosolic calcium and mediate ROS formation in cells. Herein, we summarize studies providing insights into the molecular and cellular mechanisms involved in MeHg-induced neuro degeneration with particular focus on the role of astrocytes and mitochondria. Indeed, mitochondria may be supposed to lie at the crossroads of a network of events (microtubule disorganization, $\mathrm{Ca}^{2+}$ dyshomeostasis, ROS generation) leading to neuro degeneration, although it is difficult to establish the upstream mechanisms and downstream effectors in this cascade of events.
\end{abstract}

Keywords: Methyl-mercury; Astrocytes; Neuronal Degeneration; $\mathrm{Ca}^{2+}$ homeostasis; Oxidative stress; Mitochondria

Abbreviations: BAPTA: 1,2-bis(o-aminophenoxy)ethane$\mathrm{N}, \mathrm{N}, \mathrm{N}^{\prime}, \mathrm{N}$ '-tetra acetic acid; BBB: Blood Brain Barrier; CNS: Central Nervous System; GPx: Glutathione Peroxidase; GSH: Glutathione; MeHg: Methyl Mercury; MPTP: Mitochondrial Permeability Transition Pore; NMDA: N-methyl-D-aspartate; OTC: L-2oxothiazolidine-4-carboxylic acid; ROS: Reactive Oxygen Species

\section{Introduction}

$\mathrm{MeHg}$ is a bio accumulative environmental toxicant, which has very high affinity for sulphur-containing anions, the sulfhydryl (-SH) groups on the amino acid cysteine and hence for proteins containing cysteine, forming a covalent bond. The high affinity of MeHg for thiol groups makes proteins and peptides bearing cysteines susceptible to structural and functional modification by $\mathrm{MeHg}$ in all sub cellular compartments, thus causing a range of effects at many locations throughout the body. The majority of environmental MeHg contamination occurs through the biotransformation of inorganic mercury to organic mercury in a process termed methylation. During methylation, inorganic mercury is converted into $\mathrm{MeHg}$ by microbial action, primarily in sediments of fresh and ocean waters. Thus, $\mathrm{MeHg}$ enters the aquatic food chain and is biomagnified as it accumulates in predatory fish such as swordfish, pike, and ocean tuna. Therefore, MeHg contamination in fish can be significant, and fish consumption represents an important route of human exposure [1]. MeHg, absorbed from the gastrointestinal tract, is easily transported across BBB. Once it is demethylated in the brain, elemental mercury bio accumulates in the brain tissue. Cysteine-facilitated transport of MeHg into the brain has been demonstrated, and in particular the L-type large neutral amino acid transporter [2,3] capable of mediating MeHg-cysteine uptake has been identified in astrocytes. Therefore, it has been proposed that MeHg-cysteine conjugate is the pathway whereby MeHg exerts its toxicity [4-6]. Neuronal degeneration upon $\mathrm{MeHg}$ exposure has been reported to occur either by necrosis [7-10] or apoptosis [11-15] two modes of cell death 
characterized by distinct morphological and molecular features. Several morphological and biochemical parameter hallmarks of apoptosis have been described after the acute MeHg treatment of different cell lines: neuro epithelial cells [16,17] dorsal root ganglion neurons [18], cerebellar granule cells [7,19,20], cerebrocortical neurons [12] and hippocampal cells [10]. MeHg has been shown to possess high affinity for tubulin sulfhydryl groups, to depolymerize cerebral microtubules in vitro and to inhibit microtubule assembly [21]. Microtubule fragmentation and neuronal network dissolution have been observed in cultured primary cerebellar granule neurons exposed to $\mathrm{MeHg}$ [22]. Micro tubular disruption induced by $\mathrm{MeHg}$ binding to sulfhydryl groups of peptides and resulting in the accumulation of cells in G2/M has been proposed as an important event in the development of apoptosis by MeHg in neuronal cell lines [8,14]. In the present review, we summarize studies providing insights into the molecular and cellular mechanisms involved in $\mathrm{MeHg}$ induced neuronal degeneration with particular focus on the role of astrocytes and mitochondria.

\section{MeHg accumulation in astrocytes: glutamate excitotoxicity, calcium overload, ROS production}

MeHg preferentially accumulates in astrocytes where it induces cell swelling and specifically inhibits excitatory amino acid uptake [23]. In neonatal rat cortical primary astrocyte cultures, uptake of both L-glutamate and D-aspartate is significantly reduced and efflux of both glutamate and aspartate from preloaded astrocytes are increased by $\mathrm{MeHg}$ in a doseand time-dependent fashion [24]. The consequent elevation of glutamate levels in the extracellular space may trigger or accelerate processes of excitotoxic neuro degeneration [25]. Accordingly, the co-application of non-toxic concentrations of MeHg and glutamate leads to the appearance of neuronal lesions typical of excitotoxic damage [23]. In this context, the excitatory amino acid receptors ( $\mathrm{N}$-methyl D-aspartate (NMDA) and non-NMDA-types) mediated pathways have been indicated as the main routes responsible for $\mathrm{Ca}^{2+}$ entry into cells following MeHg exposure [26]. This view is supported, at least in part, by the finding that antagonists of the NMDA receptor, including MK-801 (a non-competitive NMDA antagonist), D-2-amino5-phosphonovaleric acid (a competitive NMDA antagonist), and 7-chlorokynurenic acid (an antagonist at the glycine site associated with the NMDA receptor) can block MeHg-induced toxic effects in cerebral neuron culture $[2,27,28]$. The rising of extracellular glutamate levels is responsible for the sustained activation of glutamate receptors thus enhancing $\mathrm{Na}^{+}$influx and $\mathrm{Ca}^{2+}$ release from intracellular organelles that may trigger a biochemical cascade which promotes the ROS production [29]. Oxidative stress by itself inhibits, however, the astrocytic glutamate uptake through a direct action on the transporter proteins $[27,30]$. Endogenous glutathione (GSH) is one of the most abundant and essential thiol tripeptide for scavenging ROS [31]. The excessive formation of ROS induced by $\mathrm{MeHg}$ exposure is associated with the depleted intracellular GSH levels and can be reverted under treatment with L-2-oxothiazolidine4-carboxylic acid (OTC) [32,33]. Indeed, MeHg also inhibits astrocytic uptake of cystine and cysteine, the key precursors of GSH biosynthesis [34]. Thus, neuronal damage in response to $\mathrm{MeHg}$ most likely represents aberrant control of the extracellular milieu by astrocytes $[34,35,36]$.

\section{Mitochondria at the crossroads of a network of events leading to neuronal degeneration?}

\section{Calcium overload}

The calcium ion is known to play a critical role in cell loss in the central nervous system (CNS), and $\mathrm{Ca}^{2+}$ overload has been shown to trigger either necrotic or apoptotic cell death [29]. The biochemical mechanisms by which sustained increases in intracellular $\mathrm{Ca}^{2+}$ cause neuronal cell death include the activation of degradative enzymes, such as phospholipases, proteases and endonucleases, mitochondrial dysfunction, and perturbation of cytoskeleton organization [29]. MeHg, at low micromolar concentrations, disrupts calcium homeostasis and causes elevations in intracellular calcium in cerebellar neurons [37-40]. The $\mathrm{Ca}^{2+}$ channel blockers $\omega$-conotoxin MVIIC and nifedipine, which significantly delay MeHg-induced elevation in $\mathrm{Ca}^{2+}$ levels in this in vitro model, and the $\mathrm{Ca}^{2+}$ chelator BAPTA protect granule cells and human neuroblastoma cells from MeHg-induced cell death [38,41].

\section{Reactive oxygen species and antioxidant defenses}

ROS, such as superoxide anion, hydrogen peroxide, and hydroxyl radical are considered to be initiators of peroxidative damage. The brain is sensitive to oxidative/free radical injury and a number of studies have suggested the implication of such mechanism in the MeHg neurotoxicity [42-45]. Of particular interest, the scientific literature suggest that the targeting of thiol- and selenol-containing proteins or peptides, including $\mathrm{GSH}$, can be the primary molecular events that trigger secondary and tertiary processes that ultimately culminate in MeHginduced oxidative stress [2]. Indeed, antioxidants/oxygen radical scavengers, e.g. vitamin E, GSH, and catalase provide some degree of protection against $\mathrm{MeHg}$ deleterious effects either in vitro or in vivo [26,27,46-49]. In rat cerebellar P2 preparations, both in vitro and in vivo MeHg exposures increase the rate of ROS formation which is prevented by the iron chelator deferoxamine [42]. MeHg-induced generation of ROS seems to be a crucial event in determining progression towards damage and cell death in different cell types, including human monocytes [50], human $\mathrm{T}$ cells [51] and neurons [43,52]. The selective vulnerability of neurons to mercury has been linked to the absence or limited presence of inherent protective mechanisms such as metallothionein, GSH, heme oxygenase and other stress proteins [8] . This would explain why in adult animals, at moderate dosage of MeHg, the primary target is the CNS, although the $\mathrm{MeHg}$ concentration may be considerably higher in other tissues, such as liver and kidney. Thus, most cells have a capacity 
for resisting or repairing the damage inflicted by MeHg. If so, the targets of MeHg would be cells that cannot meet the metabolic challenge of damaged proteins and/or do not have the ability to sequester mercury via the synthesis of metallothionein or other proteins and peptides. Thus, disruption of calcium homeostasis and free radicals generation are among the detrimental effects associated with MeHg-induced toxicity $[2,8,26]$. In this scenario, mitochondria may well play a crucial role, as these organelles can act as a buffer against cytosolic calcium and mediate reactive species formation in cells $[53,54]$. Importance of mitochondria for MeHg toxicity $[26,55]$ has emerged from studies performed both in vivo [56,57] and in vitro [58,59]. In vivo exposure to $\mathrm{MeHg}$ causes its accumulation inside mitochondria followed by a series of biochemical changes in these organelles [60]. These effects are similar to those observed in studies of mitochondria respiratory chain inhibition [61].

Mitochondrial dysfunctions induced by $\mathrm{MeHg}$ include the failure of energy metabolism, the disruption of calcium homeostasis and the dissipation of the mitochondrial membrane potential, effects which lead to a mitochondrial burst of ROS production [62-64]. ROS are important mediators of damage to cell structures, including lipids and membranes, as well as proteins and nucleic acids [65]. The detrimental effects of ROS are balanced by the antioxidant action of non-enzymatic antioxidants in addition to antioxidant enzymes [65]. In vivo and in vitro experimental observations have shown that the toxic effects of MeHg are accompanied by a significant deficit of antioxidant defenses, such as the depletion of GSH and the inhibition of GSH peroxidase (GPx) activity [58,66,67]. Thus, oxidative stress is remarkably involved in MeHg-induced cytotoxicity, and the benefit of the antioxidant properties of organoselenium compounds, guanosine and flavonoids (myricetin, myricitrin, rutin) against MeHg-induced ROS generation and mitochondrial dysfunction has been demonstrated [68,69].

\section{Relationship between microtubule disorganization, $\mathrm{Ca}^{2+}$ overload and mitochondria dysfunctions}

A crucial role in cellular injury induced by MeHg has the close relationship between $\mathrm{Ca}^{2+}$ overload and mitochondria dysfunctions [26]. It is well recognized that at physiological concentration, $\mathrm{Ca}^{2+}$ is a powerful regulator of organelle metabolic activity, which acts primarily promoting ATP synthesis by stimulating crucial enzymes of Krebs cycle. On the other hand, mitochondrial changes that occur in most instances of cell death (apoptosis and necrosis) require an elevated influx of $\mathrm{Ca}^{2+}$ into matrix $[70,71]$. A series of works indicates a spectrum of mitochondrial effects, either directly or indirectly via $\mathrm{Ca}^{2+}$ overload, of MeHg both in vivo and in vitro, including alterations in complex III of the mitochondrial electron transport chain, depression of respiration and ATP production, swelling of the mitochondrial matrix, and loss of $\Delta \Psi \mathrm{m}$ with subsequent release of cytochrome c [72-74]. In myogenic cell lines, MeHg causes apoptosis via both mitochondria- and endoplasmic reticulumgenerated processes through activation of stress-activated protein kinase/c-Jun N-terminal kinase [75]. Moreover, there is a close relationship between microtubules and mitochondrial function, such as mitochondrial permeability transition pore (MPTP), thus microtubule disorganization would play an important role in mitochondrial dysfunction. In particular, stabilization and disruption of microtubule opens MPTP and depolarizes $\Delta \Psi \mathrm{m}$ in cardiac myocytes [76]. Thus, in this scenario mitochondria may be supposed to lie at the crossroads of a network of events (microtubule disorganization, $\mathrm{Ca}^{2+}$ dyshomeostasis, ROS generation) ultimately leading to neuronal degeneration, even it is difficult to establish the upstream mechanisms and downstream effectors in this cascade of events.

\section{Conclusion}

Exposure to MeHg can cause profound damage to the CNS. A number of molecular and cellular mechanisms have been identified as being the primary targets of MeHg cytotoxicity. $\mathrm{MeHg}$ preferentially accumulates in astrocytes where it induces cell swelling and specifically inhibits excitatory amino acid uptake, hence the consequent elevation of glutamate levels in the extracellular space may trigger or accelerate processes of excitotoxic neuro degeneration. MeHg exhibit high affinity for tubulin sulfhydryl groups, hence depolymerizes cerebral microtubules and inhibits microtubule assembly. Thus, micro tubular disruption has been proposed as an important event in the development of apoptosis by MeHg in neuronal cell lines. Disruption of calcium homeostasis and free radicals generation are among the detrimental effects associated with MeHg-induced toxicity. In this scenario, mitochondria may play a crucial role, as these organelles can act as a buffer against cytosolic calcium and mediate reactive species formation in cells. Mitochondrial dysfunctions induced by $\mathrm{MeHg}$ include the failure of energy metabolism, the disruption of calcium homeostasis and the dissipation of the mitochondrial membrane potential, effects which lead to a mitochondrial burst of ROS production. Remarkably, the toxic effects of MeHg are accompanied by a significant deficit of antioxidant defenses, such as the depletion of GSH and the inhibition of GPx activity. In conclusion, mitochondria are plausible active players in the network of events occurring in astrocytes and ultimately leading to neuronal degeneration.

\section{Conflict of interest}

The authors declare no conflict of interest

\section{References}

1. National Research Council (NRC) (2000) Toxicological Effects of Methyl mercury, National Academy Press, Washington, DC, USA.

2. Farina M, Aschner M, Rocha JB (2011) Oxidative stress in MeHginduced neurotoxicity. Toxicol Appl Pharmacol 256(3): 405-417.

3. Kerper LE, Ballatori N, Clarkson TW (1992) Methylmercury transport across the blood-brain barrier by an amino acid carrier. Am J Physiol 262(5pt): R761-R765.

4. Aschner M (1989) Brain, kidney and liver 203 Hg-methylmercury 
uptake in the rat: relationship to the neutral amino acid carrier. Pharmacol Toxicol 65(1): 17-20.

5. Sekine T, Cha SH, Endou H (2000) The multispecific organic anion transporter (OAT) family. Pflugers Arch 440(3): 337-350.

6. Ni M, Li X, Rocha JB, Farina M, Aschner M (2012) Glia and methylmercury neurotoxicity. J Toxicol Environ Health A 75(1617)1: 091-1101.

7. Castoldi AF, Barni S, Turin I, Gandini C, Manzo L (2000). Early acute necrosis, delayed apoptosis and cytoskeletal breakdown in cultured cerebellar granule neurons exposed to methylmercury. J Neurosci Res 59(6): 775-787.

8. Ceccatelli S, Daré E, Moors M (2010). Methylmercury-induced neurotoxicity and apoptosis. Chem Biol Interact 188(2): 301-308.

9. Miura K, Imura N (1987) Mechanism of methylmercury cytotoxicity. Crit Rev Toxicol 18(3): 161-188.

10. Tofighi R, Johansson C, Goldoni M, Ibrahim WN, Gogvadze V, et al. (2011) Hippocampal neurons exposed to the environmental contaminants methylmercury and polychlorinated biphenyls undergo cell death via parallel activation of calpains and lysosomal proteases. Neurotox Res 19(1): 183-194.

11. Bulleit RF, Cui H (1998). Methylmercury antagonizes the survival promoting activity of insulin-like growth factor on developing cerebellar granule neurons. Toxicol Appl Pharmacol 153(2): 161168.

12. Fujimura M, Usuki F, Sawada M, Rostene W, Godefroy D, et al. (2009) Methylmercury exposure down regulates the expression of Racl and leads to neuritic degeneration and ultimately apoptosis in cerebrocortical neurons Neurotoxicology 30(1): 16-22.

13. Lu TH, Hsieh SY, Yen CC, Wu HC, Chen KL, et al. (2011) Involvement of oxidative stress-mediated ERK1/2 and p38 activation regulated mitochondria-dependent apoptotic signals in methylmercuryinduced neuronal cell injury. Toxicol Lett 204(1): 71-80.

14. Miura K, Koide N, Himeno S, Nakagawa I, Imura N (1999) The involvement of microtubular disruption in methylmercury-induced apoptosis in neuronal and nonneuronal cell lines. Toxicol Appl Pharmacol 160(3): 279-288.

15. Nagashima K (1997) A review of experimental methylmercury toxicity in rats: neuropathology and evidence for apoptosis. Toxico Pathol 25(6): 624-631.

16. Faustman EM, Ponce RA, Ou YC, Mendoza MA, Lewandowski T, et al. (2002) Investigations of methylmercury-induced alterations in neurogenesis. Environ Health Perspect 110(suppl 5): 859-864.

17. Ponce RA, Kavanagh TJ, Mottet NK, Whittaker SG, Faustman EM (1994) Effects of methyl mercury on the cell cycle of primary rat CNS cells in vitro. Toxicol Appl Pharmacol 127(1): 83-90.

18. Wilke RA, Kolbert CP, Rahimi RA, Windebank AJ (2003) Methylmercury induces apoptosis in cultured rat dorsal root ganglion neurons. Neurotoxicology 24(3): 369-378.

19. Daré E, Gorman AM, Ahlbo E, Götz M, Momoi T, et al. (2001) Apoptotic morphology does not always require caspase activity in rat cerebellar granule neurons Neurotox Res 3(5): 501-514.

20. Sakaue M, Okazaki M, Hara S (2005) Very low levels of methylmercury induce cell death of cultured rat cerebellar neurons via calpain activation. Toxicology 213(1-2): 97-106.

21. Vogel DG, Margolis RL, Mottet NK (1985) The effects of methylmercury binding to microtubules Toxicol Appl Pharmacol 80(3): 473-486.
22. Castoldi AF, Coccini T, Ceccatelli S, Manzo L (2001). Neurotoxicity and molecular effects of methylmercury. Brain Res Bull 55(2): 197-203.

23. Aschner M (2000) Astrocytic swelling, phospholipase A2, glutathione and glutamate: interactions in methylmercury-induced neurotoxicity. Cell Mol Biol 46(4): 843-854.

24. Aschner M, Du YL, Gannon M, Kimelberg HK (1993) Methylmercuryinduced increases in excitatory amino acid efflux from rat primary astrocyte cultures. Brain Res 602(2): 181-186.

25. Brookes $N$ (1992). In vitro evidence for the role of glutamate in the CNS toxicity of mercury. Toxicology 76(3): 245-256

26. Roos D, Seeger R, Puntel R, Vargas Barbosa N (2012) Role of calcium and mitochondria in MeHg-mediated cytotoxicity. J Biomed Biotechnol.

27. Park ST, Lim KT, Chung YT, Kim SU (1996) Methylmercury-induced neurotoxicity in cerebral neuron culture is blocked by antioxidants and NMDA receptor antagonists. Neurotoxicology 17(1): 37-45.

28. Xu B, Xu ZF, Deng Y, Liu W, Yang HB, et al. (2012) Protective effects of MK-801 on methylmercury-induced neuronal injury in rat cerebral cortex: involvement of oxidative stress and glutamate metabolism dysfunction. Toxicology 300(3): 112-120.

29. Orrenius S, Nicotera P (1994) The calcium ion and cell death. J Neural Transm Suppl 43: 1-11.

30. Volterra A, Trotti D, Racagni G (1994) Glutamate uptake is inhibited by arachidonic acid and oxygen radicals via two distinct and additive mechanisms Mol Pharmacol 46(5): 986-992.

31. Dringen R (2000) Metabolism and functions of glutathione in brain. Prog Neurobiol 62(6): 649-671.

32. Lee YW, Ha MS, Kim YK (2001) Role of reactive oxygen species and glutathione in inorganic mercury-induced injury in human glioma cells. Neurochem Res 26(11): 1187-1193.

33. Shanker G, Syversen T, Aschner JL, Aschner M (2005) Modulatory effect of glutathione status and antioxidants on methylmercuryinduced free radical formation in primary cultures of cerebral astrocytes. Mol Brain Res 137(1-2): 11-22.

34. Shanker G, Allen JW, Mutkus LA, Aschner M (2001) Methylmercury inhibits cysteine uptake in cultured primary astrocytes, but not in neurons. Brain Res 914(1-2): 159-165.

35. Aschner M (1996) Astrocytes as modulators of mercury-induced neurotoxicity. Neurotoxicology 17(3-4):663-669.

36. Aschner M, Yao CP, Allen JW, Tan KH (2000) Methylmercury alters glutamate transport in astrocytes. Neurochem Int. 37(2-3): 199-206.

37. Marty MS, Atchison WD (1997) Pathways mediating $\mathrm{Ca}^{2+}$ entry in rat cerebellar granule cells following in vitro exposure to methylmercury Toxicol Appl Pharmacol 147(2): 319-330.

38. Marty MS, Atchison WD (1998) Elevations of intracellular Ca2+ as a probable contributor to decreased viability in cerebellar granule cells following acute exposure to methylmercury. Toxicol Appl Pharmacol 150(1):98-105.

39. Okazaki E, Oyama Y, Chikahisa L, Nagano T, Katayama N, et al. (1997) Fluorescent estimation on cytotoxicity of methylmercury in dissociated rat cerebellar neurons: its comparison with ionomycin Environ Toxicol Pharmacol 3(4): 237-244.

40. Oyama Y, Tomiyoshi F, Ueno S, Furukawa K, Chikahisa L (1994) Methylmercury-induced augumentation of oxidative metabolism in cerebellar neurons dissociated from the rats: Its dependence on 
intracellular $\mathrm{Ca}^{2+}$. Brain Res 660(1): 154-157.

41. Ndountse LT, Chan HM (2008) Methylmercury increases N-methyl-Daspartate receptors on human SH-SY 5 Y neuroblastoma cells leading to neurotoxicity. Toxicology 249(2-3): 251-255.

42. Ali SF, LeBel, CP, Bondy SC (1992) Reactive oxygen species formation as a biomarker of methylmercury and trimethyltin neurotoxicity. Neurotoxicology 13(3): 637-648.

43. Aschner M, Syversen T, Souza DO, Rocha JB, Farina M (2007) Involvement of glutamate and reactive oxygen species in methylmercury neurotoxicity. Braz J Med Biol Res 40(3): 285-291.

44. LeBel CP, Ali SF, Bondy SC (1992) Deferoxamine inhibits methylmercury-induced increases in reactive oxygen species formation in rat brain. Toxicol Appl Pharmacol 112(1): 161-165.

45. Sarafian TA, Verity MA (1991) Oxidative mechanisms underlying methylmercury neurotoxicity. Int J Dev Neurosci 9(2): 147-153.

46. Daré E, Goetz ME, Zhivotovsky B, Manzo L, Ceccatelli, S (2000) Antioxidants J811 and 17estradiol protect cerebellar granule cells from methylmercury induced apoptotic cell death. J Neurosci Res 62(4): 557-565.

47. do Nascimento JL, Oliveira KR, Crespo-Lopez ME, Macchi BM, Maués LA, et al. (2008) Methylmercury neurotoxicity and antioxidant defenses Indian J Med Res 128(4): 373-382.

48. Rush T, Liu X, Nowakowski AB, Petering, DH, Lobner D (2012) Glutathione-mediated neuroprotection against methylmercury neurotoxicity in cortical culture is dependent on MRP1. Neurotoxicology 33(3): 476-481.

49. Yonaha M, Saitoh M, Sagai M (1983) Stimulation of lipid peroxidation by methylmercury in rats. Life Sci 32(13): 1507-1514.

50. In Sug O, Datar S, Koch CJ, Shapiro IM, Shenker BJ (1997) Mercuric compounds inhibit human monocytes function by inducing apoptosis: Evidence for formation of reactive oxygen species, development of mitochondrial membrane permeability transition and loss of reductive reserve Toxicology 124(3): 211-224.

51. Shenker BJ, Guo TL, Shapiro IM (1999) Induction of apoptosis in human T-cells by methylmercury: Temporal relationship between mitochondrial dysfunction and loss of reductive reserve. Toxicol Appl Pharmacol 157(1): 23-35.

52. Yee S, Choi BH (1996) Oxidative stress in neurotoxic effects of methylmercury poisoning Neurotoxicology 17(1): 17-26.

53. Chacko BK, Srivastava A, Chang MJ, Johnson MS, Ye YZ (2009) Mitochondria- targeted ubiquinone ameliorates ethanol-induced hepatic steatosis and prevents protein nitration. Free Radical Biol Med 47: 138

54. Norenberg MD, Rao KV (2007) The mitochondrial permeability transition in neurologic disease Neurochem Int 50(7-8)9: 83-997.

55. Polunas M, Halladay A, Tjalkens RB, Philbert MA, Lowndes H, et al. (2011) Role of oxidative stress and the mitochondrial permeability transition in methylmercury cytotoxicity. Neurotoxicology 32(5): 526-534.

56. Mori N, Yasutake A, Marumoto M, Hirayama K (2011) Methylmercury inhibits electron transport chain activity and induces cytochrome c release in cerebellum mitochondria. J Toxicol Sci 36(3): 253-259.

57. Sokolowski K, Falluel-Morel A, Zhou X, DiCicco-Bloom E (2011) Methylmercury (MeHg) elicits mitochondrial-dependent apoptosis in developing hippocampus and acts at low exposure.s Neurotoxicology 32(5): 535-544.
58. Chang JY, T sai, PF (2008) Prevention of methylmercury-induced mitochondrial depolarization, glutathione depletion and cell death by 15-deoxy-delta-12, 14 prostaglandin $\mathrm{J}_{2}$. Neurotoxicoly 29(6): 1054-1061.

59. Franco JL, Braga HC, Stringari J, Missau FC, Posser T, et al. (2007) Mercurial-induced hydrogen peroxide generation in mouse brain mitochondria: protective effects of quercetin. Chem Res Toxicol 20(12): 1919-1926.

60. Denny MF, Atchison WD (1994) Methylmercury-induced elevations in intrasynaptosomal zinc concentrations: an 19F-NMR study of endogenous zinc by methylmercury J Neurochem 63(1): 383-386.

61. Mori N, Yasutake A, Hirayama K (2007) Comparative study of activities in reactive oxygen species production/defense system in mitochondria of rat brain and liver, and their susceptibility to methylmercury toxicity. Arch Toxicol 81(11): 769-776.

62. Dreiem A, Seegal RF (2007) Methylmercury-induced changes in mitochondrial function in striatal synaptosomes are calciumdependent and ROS independent. Neurotoxicology 28(4): 720-726.

63. Kang MS, Jeong JY, Seo JH, Jeon HJ, Jung KM, et al. (2006) Methylmercury-induced toxicity is mediated by enhanced intracellular calcium through activation of phosphatidylcholine specific phospholipase C. Toxicol Appl Pharmacol 216(2): 206-215.

64. Kim SH, Sharma RP (2003) Cytotoxicity of inorganic mercury in murine $\mathrm{T}$ and $\mathrm{B}$ lymphoma cell lines: involvement of reactive oxygen species, $\mathrm{Ca}^{2+}$ homeostasis, and cytokine gene expression. Toxicol In Vitro 17((4): 385-395.

65. Poli G, Leonarduzzi G, Biasi F, Chiarpotto E (2004) Oxidative stress and cell signaling. Curr Med Chem 11(9): 1163-1182.

66. Farina M, Campos F, Vendrell I, Berenguer J, Barzi M, et al. (2009) Probucol increases glutathione peroxidase-1 activity and displays long-lasting protection against methylmercury toxicity in cerebellar granule cells. Toxicol Sci 112(2): 416-426.

67. Stringari J, Nunes AK, Franco JL, Bohrer D, Garcia SC, et al. (2008) Prenatal methylmercury exposure hampers glutathione antioxidant system ontogenesis and causes long-lasting oxidative stress in the mouse brain. Toxicol Appl Pharmacol 227(1): 147-154.

68. Franco JL, Posser T, Missau F, Pizzolatti MG, Dos Santos AR, et al. (2010) Structure-activity relationship of flavonoids derived from medicinal plants in preventing methylmercury-induced mitochondrial dysfunction. Environ Toxicol Pharmacol 30(3): 272278.

69. Roos DH, Puntel RL, Santos MM, Souza DOG, Farina M, et al. (2009) Guanosine and synthetic organoselenium compounds modulate methylmercury-induced oxidative stress in rat brain cortical slices: involvement of oxidative stress and glutamatergic system. Toxicol In Vitro 23(2): 302-307.

70. Duchen MR (2000) Mitochondria and calcium: from cell signaling to cell death. J Physiol 529: 57-68.

71. Kruman II, Mattson MP (1999) Pivotal role of mitochondrial calcium uptake in neural cell apoptosis and necrosis. J Neurochem 72(2): 529-540.

72. Bondy SC, McKee M (1991) Disruption of the potential across the synaptosomal plasma membrane and mitochondria by neurotoxic agents. Toxicol Lett 58(1): 13-21.

73. Limke TL, Atchison WD (2002) Acute exposure to methylmercury opens the mitochondrial permeability transition pore in rat cerebellar granule cells. Toxicol Appl Pharmacol 178(1): 52-61. 
74. Shenker BJ, Pankoski L, Zekavat A, Shapiro IM (2002) Mercuryinduced apoptosis in human lymphocytes: caspase activation is linked to redox status. Antioxid Redox Signal 4(3): 379-389.

75. Usuki F, Fujita E, Sasagawa N (2008) Methylmercury activates ASK1/JNK signaling pathways, leading to apoptosis due to both mitochondria- and endoplasmic reticulum (ER)-generated processes in myogenic cell lines. Neurotoxicology 29(1): 22-30.

76. Kumazawa A, Katoh H, Nonaka D, Saotome M, Urushida T, et al. (2011) Microtubule stability is related to mitochondrial function in rat ventricular myocytes 